\title{
Finite Size Effects on the QCD Spectrum Revisited*
}

\author{
MIMD Lattice Calculation (MILC) Collaboration - C. Bernard, ${ }^{a}$ T. A. DeGrand, ${ }^{\mathrm{b}}$ C. DeTar, ${ }^{\mathrm{c}}$ Steven \\ Gottlieb, ${ }^{\text {de }}{ }^{\dagger}$ A. Krasnitz, ${ }^{\text {d }}$ R. L. Sugar, ${ }^{f}$ and D. Toussaint ${ }^{g}$

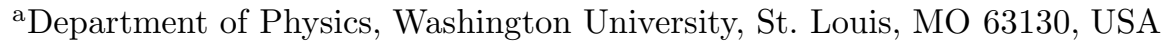 \\ ${ }^{\text {b} P h y s i c s ~ D e p a r t m e n t, ~ U n i v e r s i t y ~ o f ~ C o l o r a d o, ~ B o u l d e r, ~ C O ~ 80309, ~ U S A ~}$ \\ ${ }^{\mathrm{c}}$ Physics Department, University of Utah, Salt Lake City, UT 84112, USA \\ ${ }^{\mathrm{d}}$ Department of Physics, Indiana University, Bloomington, IN 47405, USA \\ ${ }^{\text {e}}$ Department of Physics, Brookhaven National Laboratory, Upton, NY 11973, USA \\ ${ }^{\mathrm{f}}$ Department of Physics, University of California, Santa Barbara, CA 93106, USA \\ ${ }^{\mathrm{g}}$ Department of Physics, University of Arizona, Tucson, AZ 85721, USA
}

\begin{abstract}
We have continued our study of finite size effects in the QCD spectrum on lattices ranging in size from $8^{3} \times 24$ to $16^{3} \times 24$. We have increased our statistics for quark mass $a m_{q}=0.025$ for the smallest lattice size. In addition, we have studied quark mass 0.0125 for lattice sizes $12^{3} \times 24$ and $16^{3} \times 24$. These lattice sizes correspond to a box 1.8-3.6 fm on a side when the rho mass at zero quark mass is used to set the scale. We discuss the nucleon to rho mass ratio at a smaller value of $m_{\pi} / m_{\rho}$ than previously studied with two dynamical flavors.
\end{abstract}

\section{MOTIVATION}

For some time, it has been recognized that there can be substantial finite size effects in the hadron spectrum if the box size is too small. This was seen very early on in the quenched approximation[1]. In the first dynamical fermion spectrum calculations done by some of us, we used two different volumes in each case to try to assess the magnitude of this effect[2]. More recently, there have been calculations at weaker coupling over a wider range of lattice volumes showing very dramatic finite volume effects 3]. Our approach is to work with stronger coupling so that we can go to large physical volumes. We do this in the hope that by precisely evaluating the finite volume effects where they are small, we can set the physical box size for weaker coupling calculations where it would be very costly to go to such large physical volumes. That is, we want to go to a very

\footnotetext{
${ }^{*}$ Presented by S. Gottlieb

${ }^{\dagger} \mathrm{BNL}$ is supported under DOE Contract DE-AC02$76 \mathrm{CH} 00016$.
}

large physical volume and directly demonstrate that our largest volumes are more than adequate. We thus determine which physical volume is sufficient to achieve a 1 or $2 \%$ accuracy, and suggest that physical volume as the minimum for weaker coupling calculations. We would also like to make

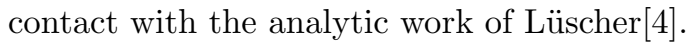

Another advantage of stronger coupling is that we can go to smaller values of $m_{\pi} / m_{\rho}$ than is currently possible at weaker coupling. This light quark mass region is of interest because $m_{N} / m_{\rho}$ should decrease in the chiral limit and because this is where we should start to see the effects of rho decay. It is well known that the $m_{N} / m_{\rho}$ ratio has always come out too large in lattice calculations. It is possible that only when we make both the quark mass lighter and the lattice spacing smaller will we approach the observed value. Approaching the chiral limit at accessible lattice spacings strikes us as more relevant to the eventual extrapolation than decreasing the lattice spacing at say $m_{\pi} / m_{\rho}=0.5$. 
Table 1

Summary of Staggered Spectrum Runs

\begin{tabular}{lclr}
\hline$a m_{q}$ & $6 / g^{2}$ & size & length \\
\hline 0.025 & 5.445 & $8^{3} \times 24$ & 1780 \\
0.025 & 5.445 & $8^{3} \times 24$ & 1554 \\
0.025 & 5.445 & $10^{3} \times 24$ & 1392 \\
0.025 & 5.445 & $12^{3} \times 24$ & 1036 \\
0.025 & 5.445 & $16^{3} \times 24$ & 1556 \\
0.0125 & 5.415 & $12^{3} \times 24$ & 974 \\
0.0125 & 5.415 & $12^{3} \times 24$ & 282 \\
0.0125 & 5.415 & $16^{3} \times 24$ & 1220 \\
\hline
\end{tabular}

\section{SPECTRUM CALCULATION}

The couplings that we use correspond to the finite temperature crossover for $N_{t}=6$. Since the smallest lattice we use has $N_{s}=8$, we have no worry that we are suffering from "spatial deconfinement." Our minimum lattice size is about $1.75 \mathrm{fm}$. We use two quark masses, $a m_{q}=0.025$ and 0.0125 , which we will refer to as the heavier and lighter mass. (For details, see Table 1.)

All of our masses come from fits with two particles. For the pion channel, we assume two pseudoscalars. For all the other channels, we assume two particles of opposite parity. To see the finite size effects, it is important for us to find the optimal set of fits. For all of our fits, we use the full covariance matrix with 20 time units blocked together to calculate the covariance matrix. We fit from $D_{\min }$ to the center of the lattice for all particles but the nucleon. Due to antiperiodic boundary conditions the nucleon propagator should vanish on the center plane, so we ignore its value there. For the heavier quark mass, we have four lattice volumes, and we choose the fit according to the combined confidence level of all four fits. The rho combined confidence level is $0.25,0.20$ and 0.12 , for $D_{\min }=4,5$ and 6 , respectively. Last year we presented pion masses based on single particle fits. For $D_{\min }=7$ and 8 , they both have combined confidence levels of 0.27 . However, the two particle fits with $D_{\min }=2$ that we report here have a combined confidence level of 0.48 . More details of the combined confidence levels are found in Ref. 河, which contains a complete table of masses. For the heavier (lighter)
Table 2

Hadron Finite Size Effects

\begin{tabular}{lll}
\hline & 1991 Result & 1992 Result \\
\hline$\pi\left(N_{s}=8\right)$ & $0.4542(15)$ & $0.4529(7)$ \\
$\pi\left(N_{s}=16\right)$ & $0.4483(6)$ & $0.4488(4)$ \\
Difference & $0.0059(16)$ & $0.0041(8)$ \\
Significance & $3.7 \sigma$ & $5.1 \sigma$ \\
Percentage & $1.3 \%$ & $0.9 \%$ \\
\hline$\rho\left(N_{s}=8\right)$ & $0.947(18)$ & $0.949(12)$ \\
$\rho\left(N_{s}=16\right)$ & $0.918(5)$ & $0.918(4)$ \\
Difference & $0.029(19)$ & $0.031(13)$ \\
Significance & $1.5 \sigma$ & $2.4 \sigma$ \\
Percentage & $3.2 \%$ & $3.4 \%$ \\
\hline$N\left(N_{s}=8\right)$ & $1.482(55)$ & $1.456(22)$ \\
$N\left(N_{s}=16\right)$ & $1.380(8)$ & $1.375(8)$ \\
Difference & $0.102(56)$ & $0.081(23)$ \\
Significance & $1.8 \sigma$ & $3.5 \sigma$ \\
Percentage & $7.4 \%$ & $5.9 \%$ \\
\hline
\end{tabular}

quark mass, we use $D_{\min }=4(3)$ for all particles other than the pion. For the pion, we use $D_{\min }=2$ for both masses.

\section{FINITE SIZE EFFECTS}

For the heavier quark mass, we presented results last year at Tsukuba [6]. By increasing the statistics on our smallest lattice, we have been able to increase the statistical significance of the difference in masses between the smallest and largest volume. For the lighter mass, we only have simulations for the two larger volumes. Here, we can only bound the size of the effect. The effect should be larger with smaller quark mass, but we don't have a comparable range of volume.

In Table 2, we compare our 1991 results with the new fits and increased statistics. The pion mass is actually a little smaller for $N_{s}=8$ than before; however, the errors have decreased, so the finite size effect is smaller but statistically more significant. Most of the effect seems to occur between $N_{s}=8$ and 10. In Fig. 1, we show the pion mass as a function of $N_{s}$.

Turning now to the rho, we see that there is an effect slightly larger than three percent between the smallest and largest lattice. For the nucleon, we have the largest effect, almost six percent. Be- 
- Figure 2. Rho mass as a function of lattice size

Figure 1. Pion mass as a function of lattice size

cause of the reduced errors, the significance of this effect has gone from $1.8 \sigma$ to $3.5 \sigma$. The masses are plotted as a function of $N_{s}$ in Figs. 2 and 3. Results for the $\pi_{2}$ and $\rho_{2}$ may be found in Ref. [5].

How large must our box be to get within one or two percent of the infinite volume limit? It appears from our results that $N_{s}=12$ would suffice. To translate this into a physical size, we set the lattice spacing by extrapolating the rho mass to zero quark mass and assuming that it has its physical value. In this way, we find that $m_{\rho}\left(m_{q}=0\right)=0.887(38)$ and the lattice spacing is $0.227 \mathrm{fm}$. Our range $8 \leq N_{s} \leq 16$ corresponds to sizes of 1.8 to $3.6 \mathrm{fm}$, and we are suggesting that a box size of $2.7 \mathrm{fm}$ would be adequate for the quark masses studied here (corresponding to $\left.m_{\pi} / m_{\rho} \approx 0.4\right)$. Our calculations should be compared with those done at $6 / g^{2}=5.7$ where $m_{\rho}\left(m_{q}=0\right)=0.340(16)$, and the lattice spacing
Figure 3. Nucleon mass vs. $N_{s}$

determined from the rho mass is $0.087 \mathrm{fm}$. Here $N_{s}$ ranges from 8 to 20 and corresponds to sizes from 0.7 to $1.7 \mathrm{fm}$ [3:]7. Recently, results have become available for $N_{s}=32$ which corresponds to a size of $2.8 \mathrm{fm}$, but only for $m_{\pi} / m_{\rho}=0.72[8]$.

\section{EDINBURGH PLOT}

Our Edinburgh plot is shown in Fig. 4. To calculate the error on the ratio of hadron masses, we have simply added the individual percentage errors. This naive procedure probably overestimates the size of the error since the hadrons' 
masses are all correlated. Nevertheless, we have quite high precision, particularly for the largest volume (octagon for $m_{q}=0.025$ and fancy square for $\left.a m_{q}=0.0125\right)$. Last year, we had some preliminary results for $a m_{q}=0.0125$ on our smaller lattice that indicated an improvement in $m_{n} / m_{\rho}$ as the quark mass is decreased. On our larger lattice, the nucleon mass has increased, and the rho mass has decreased. The mass ratio thus increased by about 2 standard deviations when the volume was increased. Unfortunately, this puts the nucleon to rho mass ratio back to about 1.5. At this point, we must conclude that a light quark mass at this strong coupling is not sufficient to cause the nucleon to rho mass ratio to decrease toward its observed value. It seems essential to decrease the lattice spacing. (See Ref. [9].)

- Figure 4. Edinburgh plot for this calculation

\section{CONCLUSIONS}

We have explored finite size effects by looking at large lattices with high statistics, though at a fairly strong coupling. We see significant effects for the pi, rho and nucleon. The box sizes used here ranged from about 1.8-3.6 fm. Much of the observed effect occurs between 1.8 and $2.3 \mathrm{fm}$. A size of $2.7 \mathrm{fm}$ seems adequate for our higher mass to get results within 1 to 2 percent. For the lighter quark mass, we really should explore smaller and larger volumes. At the $2 \%$ level, we cannot see any finite volume effect between $N_{s}=12$ and 16 .

There seems to be little improvement in $m_{N} / m_{\rho}$ at this coupling as we lower the quark mass. Probably we need both weak coupling and light quarks to reproduce the observed ratio.

It is important to study other quark masses and to try to make quantitative contact with analytic predictions. Very high statistics will be required. It is hoped that careful work at stronger coupling will set the physical box size for the most aggressive calculations at weak coupling.

\section{Acknowledgements}

This work was supported by both the U.S. Department of Energy and the National Science Foundation. Computations were done on Intel iPSC/860 supercomputers at the San Diego Supercomputer Center, the NASA Ames Laboratory and the SSC Laboratory.

\section{REFERENCES}

1. P. Hasenfratz and I. Montvay, Phys. Rev. Lett. 50 309, 1983; H. Lipps, G. Martinelli, R. Petronzio and F. Rapuano, Phys. Lett. 126B 250, 1983; R. Gupta and A. Patel, Phys. Lett. 124B 94, 1983; C. Bernard, T. Draper, K. Olynyk and M. Rushton, Nucl. Phys. B220 508, 1983.

2. S. Gottlieb, W. Liu, D. Toussaint, R. L. Renken, and R. L. Sugar, Phys. Rev. D 38 $2245,1988$.

3. M. Fukugita, H. Mino, M. Okawa, and A. Ukawa, Nucl. Phys. B (Proc. Suppl.) 20 376, 1991; Phys. Rev. Lett. 68 761, 1992.

4. M. Lüscher, Comm. Math. Phys. 104 177, 1986.

5. C. Bernard et al, Finite Size Effects in the QCD Spectrum with Two Flavors, preprint IUHET-232.

6. C. Bernard et al, Nucl. Phys. B (Proc. Suppl.) 26 262, 1992.

7. F. Brown et al, Phys. Rev. Lett. 67 1062, 1991. 
8. See the talk by W. Schaffer in this volume.

9. See the talk by A. Ukawa in this volume. 